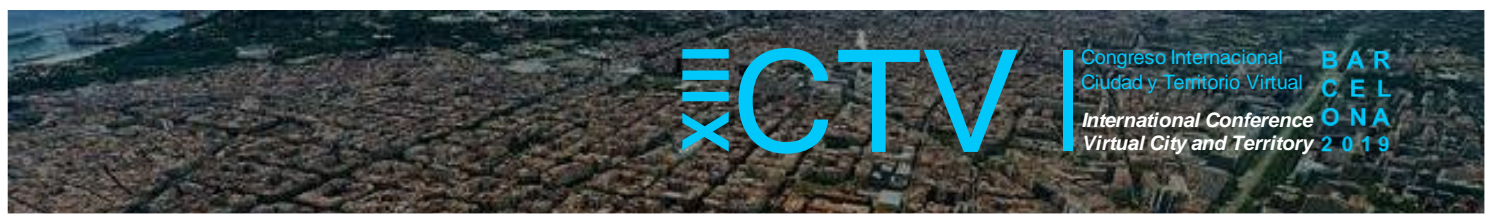

\title{
CENTRO CÍVICO VIL·LA URÀNIA, UN EDIFICIO nZEB
}

Bendicho, Pasqual ${ }^{1 *}$; Camallonga, Marc ${ }^{2}$; Pagès, Jordi ${ }^{3}$ y Olmo, Yolanda ${ }^{4}$

Remisión inicial: 2019-06-16; Remisión definitiva: 2019-20-20; Publicación: 2019-12-21

Citación: Bendicho, P. et al. (2019). Centro cívico Vil·la Urània, un edificio nZEB. En XIII CTV 2019 Proceedings: XIII International Conference on Virtual City and Territory: "Challenges and paradigms of the contemporary city": UPC, Barcelona, October 2-4,2019. Barcelona:CPSV, 2019, p. 8643. E-ISSN2604-6512.DOI http://dx.doi.org/10.5821/ctv.8643

\section{Resumen}

El Centro Cívico Vil·la Urània en Barcelona es un edificio construido bajo parámetros de consumo energético casi nulo (nZEB). Es decir, un edificio que consume poca energía, muy por debajo de un edificio de las mismas características y que parte de la energía consumida se produce en el mismo edificio o cerca de él.

Un equipamiento de $3200 \mathrm{~m} 2$ que destaca por el uso de espacios intermedios, climatizados de forma natural, cerrados por una gran fachada formada por varios filtros superpuestos que se adaptan a las condiciones exteriores, proporcionando una fachada vegetal que acompaña a los usuarios en todas sus actividades. Una planta fotovoltaica en la azotea, pozos geotérmicos esparcidos por el solar y un eficiente tratamiento del ciclo del agua aportan energía limpia al conjunto edificado.

La ampliación se concibe como un edificio alto y estrecho, orientado a Sur-este con una gran galería. Un invernadero adosado, que climatiza de forma pasiva las zonas de encuentro y de actividades informales, así como las circulaciones del edificio. Este espacio intermedio funciona como un invernadero en invierno y como un umbráculo en verano, y actúa como colchón térmico al separar las zonas climatizadas del exterior, reduciendo la demanda energética del edificio. La fachada se adapta de forma automática a las condiciones exteriores. Sensores de temperatura interior actúan sobre la fachada de vidrio, abriéndola completamente cuando es necesario. Sondas exteriores miden la radiación solar actuando sobre las persianas replegables en verano. La plantación interior formada por diferentes especies proporciona una agradable sensación de frescor en verano, mientras que en invierno reduce su volumen para permitir captar la radiación solar.

\section{Abstract}

Vil-la Urània Civic Center is a building designed under nearly Zero Energy building parameters (nZEB). A building with low environmental impact and reduced energy consumption, and that produces part of the energy it needs at the building itself or close to it.

An equipment, with more than $3200 \mathrm{~m} 2$, stands out for the use of intermediate spaces, naturally heated, closed by a large facade formed by several overlapping filters that adapt to the external conditions, providing a vegetal facade that accompanies the users in all their activities. A photovoltaic production plant, geothermal heat pump systems split over the site plot and an efficient water-cycle management, brings clean energy to the built complex.

The extension is conceived as a tall and narrow building, oriented to Southeast with a large gallery, a semidetached greenhouse, a passive system to warm and refresh the meeting areas and informal activities as well as the circulation of the building. This intermediate space works as a winter greenhouse and as a shaded house in summer, and acts as a thermal mattress by separating the heated areas from the outside, reducing the energy demand of the building. The facade adapts automatically to outdoor conditions. Indoor temperature sensors act on the glass facade, opening it completely when necessary. Outside sensors measure solar radiation by acting on the folding shutters in summer. The inner plantation formed by different species provides a pleasant sensation of freshness in summer, while in winter reduces its volume to allow to capture the solar radiation.

Palabras Clave: nZEB, Espacios Intermedios; LEED Platinum; Fachada Dinámica

Key words: nZEB; Intermediate Spaces; LEED Platinum; Dynamic Façade

\footnotetext{
${ }^{1}$ SUMO arquitectes; Profesor asociado del Departamento de Representación Arquitectónica ETSAB-UPC; ${ }^{2}$ SUMO arquitectes; Profesor asociado del Departamento de Proyectos Arquitectónicos ETSAV-UPC; ${ }^{3}$ SUMO arquitectes; Profesor asociado del Departamento de Tecnología de la Arquitectura ETSAB-UPC. * Correo de contacto: pasqual@sumo-arquitectes.com
} 


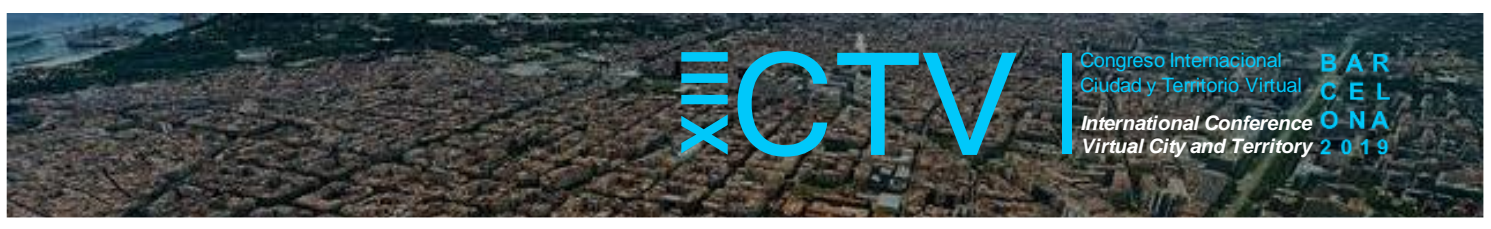

\section{Introducción. Vil·la Urània, una oportunidad}

La Vil.la Urània es una pequeña residencia de finales del siglo XIX que fue hogar del reconocido astrónomo Josep Comas i Solà, en el distrito de Sarriá-Sant Gervasi en Barcelona. La re-densificación del barrio dejó el edificio y el pequeño jardín circundante encajonado entre dos grandes medianeras.

En el año 2013 el Ayuntamiento de Barcelona a través de un concurso público promovió el Complejo de equipamientos Vil-la Urània, un edificio de nueva planta en el solar contiguo a la villa que debía incorporar el edificio pre existente.

Las bases del concurso definían el programa funcional y el planeamiento asociado, pero en ningún caso se requería un bajo consumo energético de forma explícita.

Partiendo de una clara concepción inicial, la orientación y las características inherentes del solar resultaron ser parte de la propuesta. Un estudio energético basado en modelos y simulaciones energéticas sobre la captación pasiva a través de la fachada acristalada a lo largo de todo el año fue crucial en la metodología de desarrollo y validación de la propuesta.

Una vez ejecutado el edificio se ha realizado una monitorización del mismo a lo largo de todo un año. Dicho seguimiento ha permitido validar las hipótesis simuladas en fase de proyecto y ajustar el sistema al usuario final para asegurar el confort térmico en todas y cada una de las estaciones del año.

El edificio dispone de certificación energética A y certificación LEED Platinum del edificio terminado.

Figura 1. Génesis del proyecto

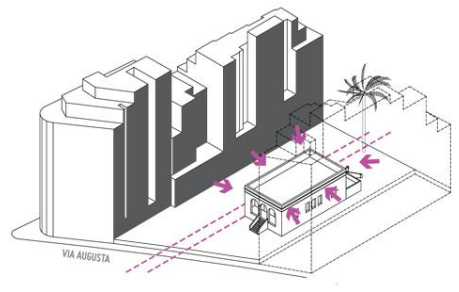

VIL.LA URÀNIA Y SU EMPLAZAMIENTO PUNTO DE PARTIDA

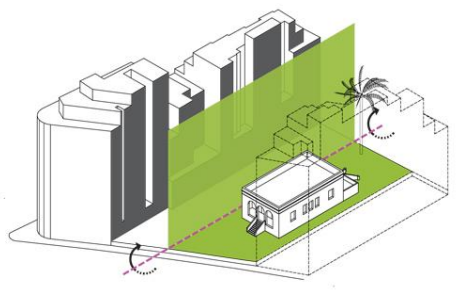

JARDIN VERTICAL - ILUSIONISMO

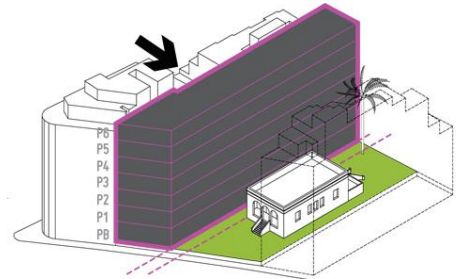

VOLUMETRIA MÁXIMA PERMITIDA

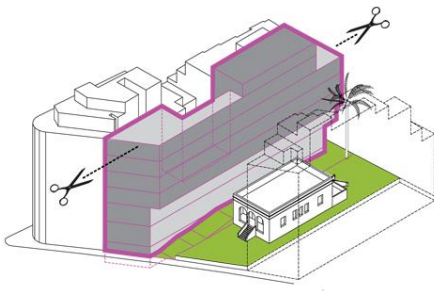

ADAPTACIÓN DELA VOLUMETRIA A SU ENTORNO

Fuente: SUMO arquitectes SLP.

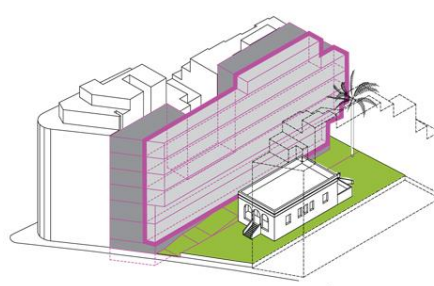

SEGREGACIÓN DE GIRCULACIONES ESREGACION DE CIRCULACIONES

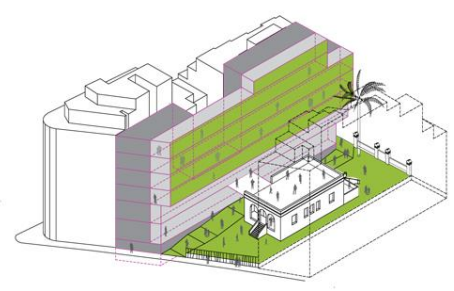

IUEVO COMPLEJO DE EQUIPAMIENTOS VILLLA URÀNIA 


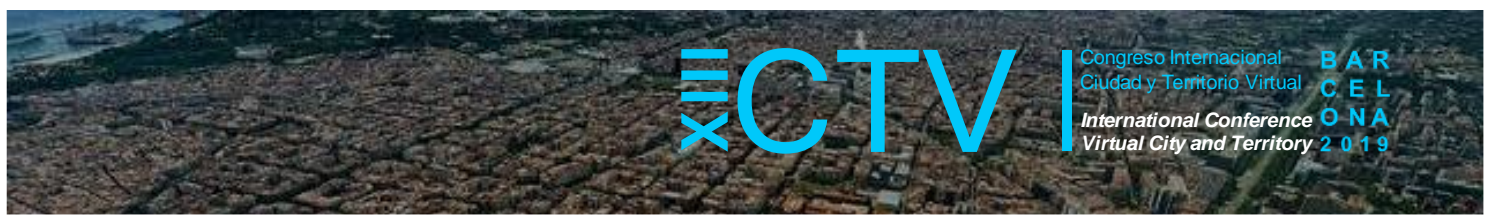

\section{Reducción de la demanda energética}

La clave en un edificio nZEB consiste en reducir al máximo la demanda energética de su puesta en marcha. El nuevo edificio, alargado y estrecho, cuenta con una fachada principal muy alargada orientada a sur-este. En ella se concentran las circulaciones del edificio. Un espacio entendido como una extensión de la calle dentro del edificio. Una fachada galería cubierta por lamas de cristal. Un espacio intermedio que en invierno se climatiza de forma pasiva mediante la radiación solar que recibe durante gran parte del día. La losa de hormigón armado, con una alta inercia, actúa de acumulador energético y disipa lentamente el calor acumulado cuando el sol se va. Toda esta zona se climatiza gracias al efecto invernadero. El salto térmico interior-exterior se reduce sustancialmente de modo que los sistemas activos en los espacios climatizados trabajan menos, reduciendo sustancialmente la demanda energética. En verano la fachada se abre completamente y las persianas replegables generan sombra a modo de umbráculo. La temperatura del aire es la misma tanto en el interior como en el exterior, pero gracias a la evaporación de las plantas aumenta la humedad entorno el espacio intermedio, aumentando la sensación de frescor y menor temperatura (Figura 2).

Figura 2. Sección por el espacio intermedio en verano y en invierno

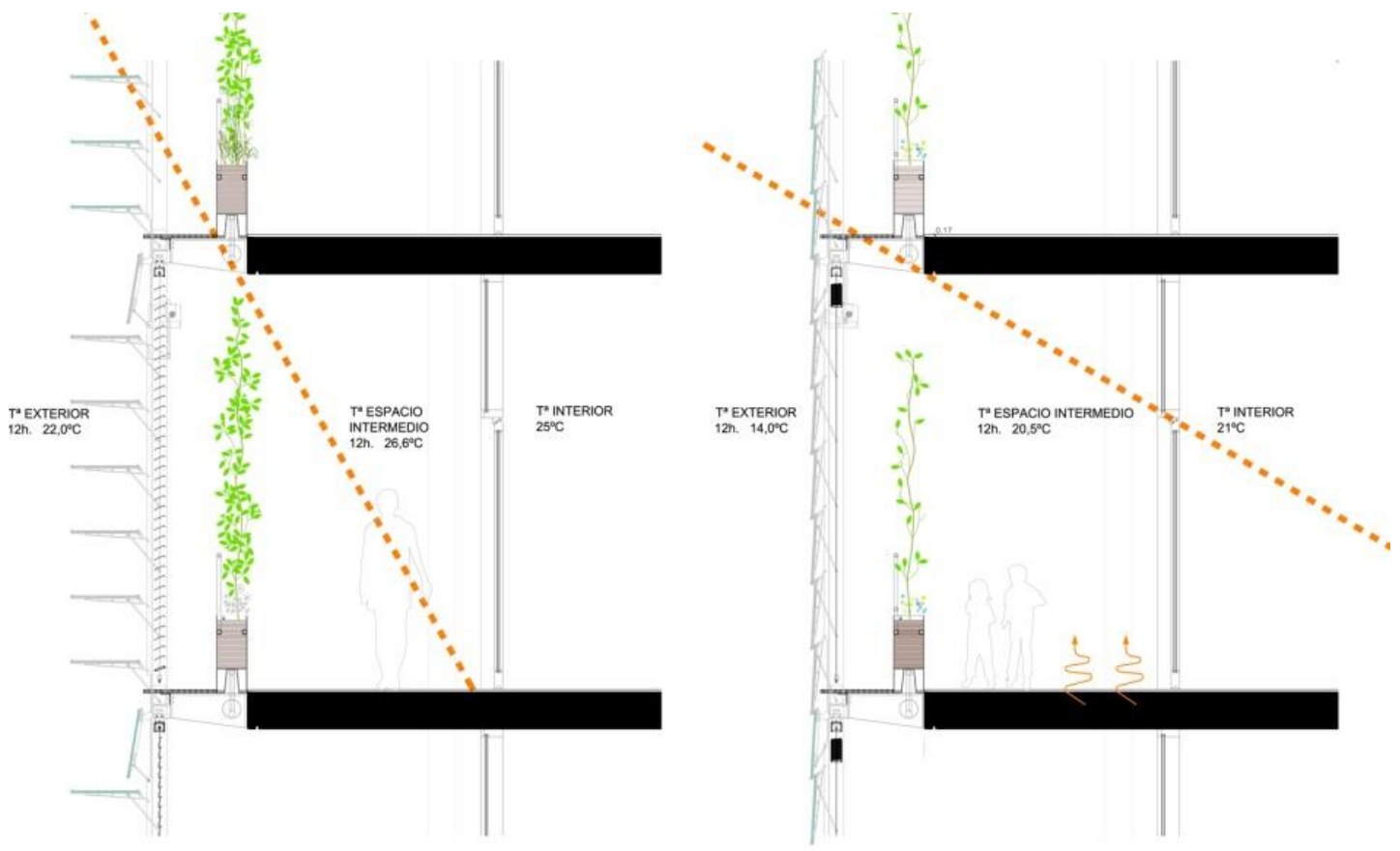

Fuente: SUMO arquitectes SLP.

El resto de la envolvente del edificio se ha diseñado para conseguir una transmitancia térmica baja, minimizar los puentes térmicos y un nivel alto de estanqueidad (Figura 3) Cubierta U:0,23 $\mathrm{W} / \mathrm{m} 2 \mathrm{~K}$, Fachada U:0,26-0,31 W/m2K, Muros enterrados U:0,26 W/m2K, Solera U:0,44 W/m2K. Los acristalamientos se han seleccionado dependiendo de su ubicación concreta: Acristalamientos entre espacio Interior y el Exterior: Ug:1,3 W/m2K TL:70\% g:0,41; Acristalamientos entre espacio Interior y el Intermedio: Ug:1,5 W/m2K TL:78\% g:0,65; Acristalamientos entre espacio Intermedio y el Exterior: Ug:5,4 W/m2K TL:80\% g:0,82 y Carpintería de madera Um:2W/m2K. 


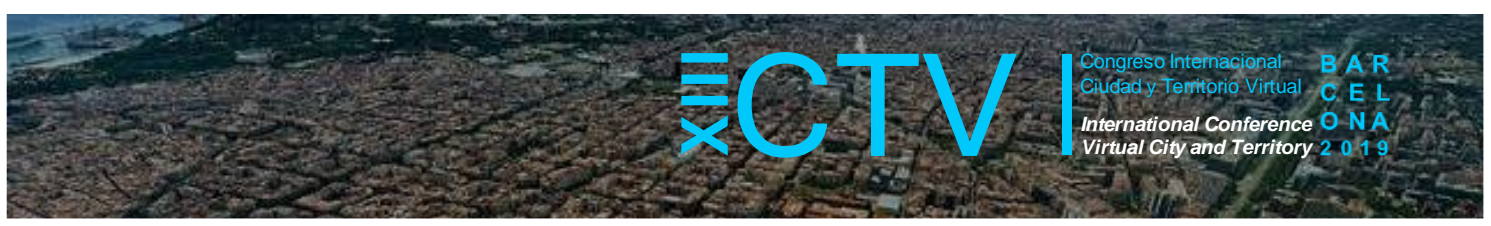

El objetivo es que el edificio esté muy bien aislado para evitar pérdidas o ganancias de energía segun sea invierno o verano.

Figura 3. Transmitancias de envolventes

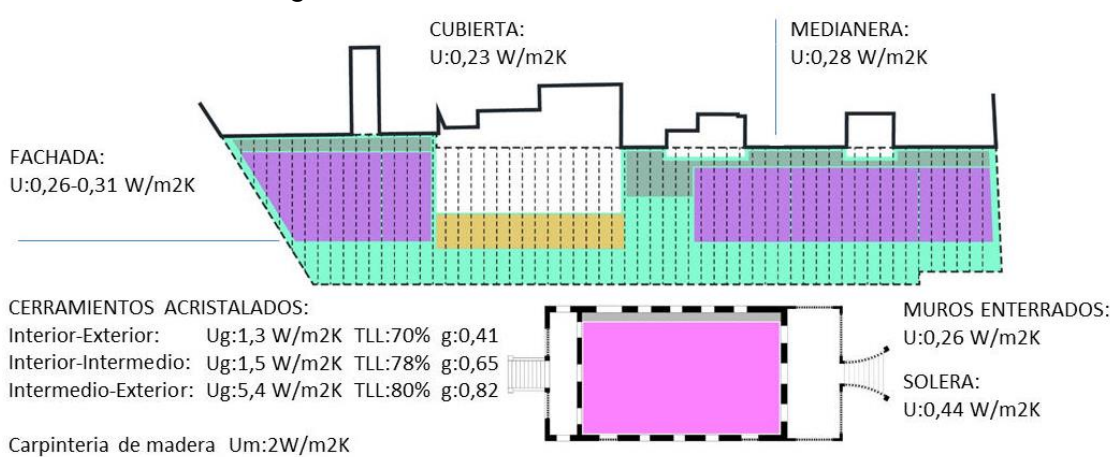

Fuente: SUMO arquitectes SLP.

\section{Sistemas activos de alta eficiencia}

Para reducir el consumo de energía se han escogido sistemas activos de alta eficiencia. El edificio dispone de una bomba de calor geotérmica de $200 \mathrm{~kW}$ con 11 pozos de $100 \mathrm{~m}$ de profundidad (100kW) que se combina con un evaporador remoto de $160 \mathrm{~kW}$ para los momentos punta y cuando la temperatura exterior es más favorable. Las salas disponen de inductores (vigas frías) y un sistema de aire primario (Figura 4). El aire primario se impulsa a través de las vigas frías dando calor o frio según sea necesario. Recuperadores de calor de alta eficiencia controlados por sensores de presencia y de $\mathrm{CO}_{2}$ permiten realizar la renovación de aire solo cuando es preciso.

La iluminación es LED y en cubierta hay una planta de producción fotovoltaica con 19kWpic instalados (Figura 5). Un depósito enterrado de 20.000 Lermite recoger agua de lluvia y usarla en un circuito cerrado para el riego de la plantación del edificio, cerrando eficientemente el ciclo del agua.

Figuras 4 y 5 . Sistemas activos (Vigas Frías) y Esquemas de Instalaciones
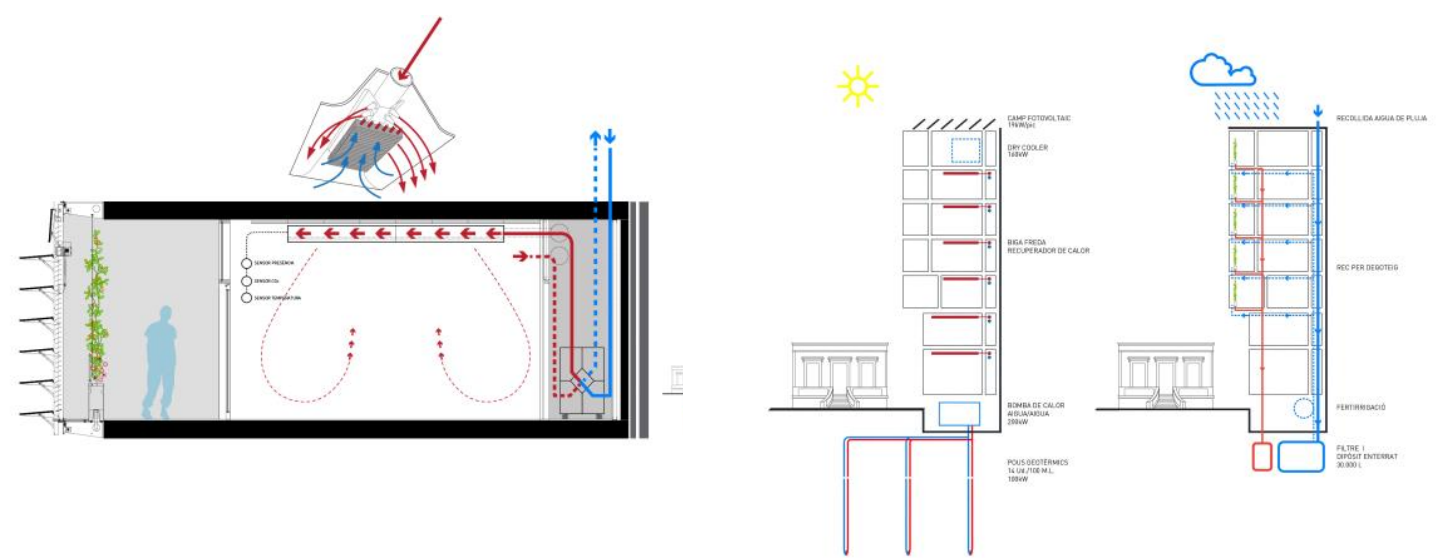

Fuente: SUMO arquitectes SLP. 


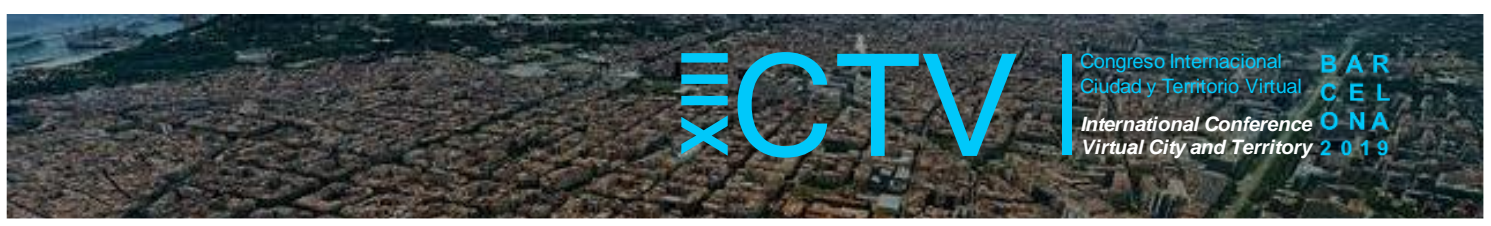

Todo el edificio esta automatizado mediante un sistema informático centralizado que puede ser controlado de forma remota. Dispone de un completo sistema de monitorización. También se diseñó un interfaz a medida, de forma que parte de los datos: consumo eléctrico, producción fotovoltaica, temperaturas etc. se pueden ver a tiempo real en unos monitores ubicados en el Centro Cívico para concienciar a los usuarios.

\section{Materiales de bajo impacto ambiental}

Para la ejecución se seleccionaron materiales y sistemas constructivos con un contenido de COV (compuestos orgánicos volátiles) muy bajos. Se dio prioridad a materiales naturales de poca elaboración y con poco tratamiento, dejándolos vistos.

A su vez la mayoría de materiales son de bajo impacto ambiental, rápidamente renovables y de origen reciclado.

El uso de la madera está muy presente en el nuevo edificio. Carpintería de madera, armarios, puertas, muros cortina mixtos de aluminio y madera. Este material además de reducir la huella ecológica permite la industrialización en taller y montar posteriormente en obra de modo que se reducen sustancialmente los plazos de ejecución.

La estructura se resolvió con muros y losas de hormigón y pilares metálicos montados previamente en taller. El resto del edificio se basa en sistemas constructivos en seco y altamente industrializados. En definitiva, un proceso constructivo de rápida puesta en obra y de ejecución muy eficiente.

Dichos materiales son altamente reciclables y podrán ser aprovechados cuando el edificio llegue al final de su vida útil.

\section{Normativa legal y el papel de la administración}

La directiva europea 2010/31/UE estableció que antes del 1 de junio de 2015 cada Estado miembro concretaría la definición exacta de los parámetros que debería cumplir un edificio de consumo energético casi nulo. Además de eso, todos los edificios de nueva planta a partir de enero del 2021 deberían ser nZEB.

Pocos meses antes de la convocatoria del concurso se aprobó el Real Decreto 235/2013 (CTE 2013) que regulaba el procedimiento de la certificación de eficiencia energética de edificios en su disposición adicional segunda. A fecha de hoy el CTE sigue siendo el único referente legal en España para establecer los requisitos mínimos en cuanto a eficiencia energética se refiere sin concretar los parámetros exactos que debe cumplir un edificio para considerarlo nZEB.

EI ICAEN, Institut Català de l'Energia ha establecido unos parámetros orientativos para un edificio nZEB en una zona climática C2 como podría ser en Barcelona:

- Rango de demanda Energética: Ahorro del 35\% de demanda respecto al edificio de referencia

- Porcentaje de contribución de energías renovables: $\geq 70 \%$

- Rango de consumo de energía primaria y emisiones de $\mathrm{CO}_{2}$ Clase A

El Centro Cívico Vil-la Urània tiene un ahorro del $45 \%$ respecto al edificio de referencia, un

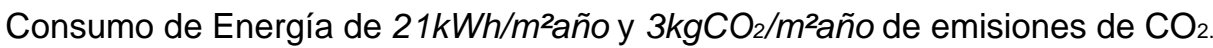




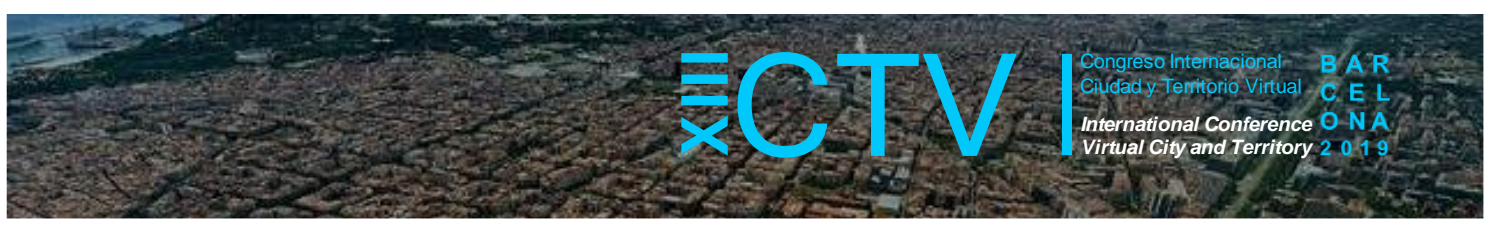

En su día, BIMsa, la empresa municipal al cargo del proyecto solicitó un estudio energético que validase la propuesta planteada. Además, a modo de prueba propusieron incorporar en el proyecto una auditoría energética externa. Se optó por una certificación LEED. En fase de proyecto se llegó hasta una certificación Platinum, el máximo en su rango de puntuación.

En la licitación de las obras, la certificación LEED resultó un punto de distinción entre las constructoras licitadoras, como sello de calidad y responsabilidad constructiva.

El compromiso por parte de la empresa adjudicataria del contrato a cumplir la certificación LEED Platinum resultó muy útil en el proceso de elección de la calidad de los materiales en obra.

\section{Conclusiones}

El Centro Cívico Vil-la Urània se ha convertido en un edificio de referencia para las administraciones del área metropolitana de Barcelona y ha despertado gran interés por la simplicidad de soluciones, por sus bajos consumos y responsabilidad sostenible.

Ha sido galardonado con diversos premios y menciones como el Green Solution Awards 2017 en la categoría Smart Building entre otros. El bajísimo consumo energético del edificio queda prácticamente compensado con la energía limpia que genera el edificio. Ha resultado ser una herramienta pedagógica de gran impacto social donde los usuarios entienden en un ejemplo construido conceptos abstractos de forma clara y sencilla.

Por otro lado, la administración empieza a entender estos edificios como un nuevo paradigma donde todo el mundo y el planeta sale ganando, pero que implica una nueva forma de afrontar los proyectos, el control y mantenimiento de dichos inmuebles. Las soluciones pueden ser escalonadas y posiblemente extrapolables a otros edificios, pero partiendo siempre del entendimiento y profundo análisis inicial de las posibilidades del lugar.

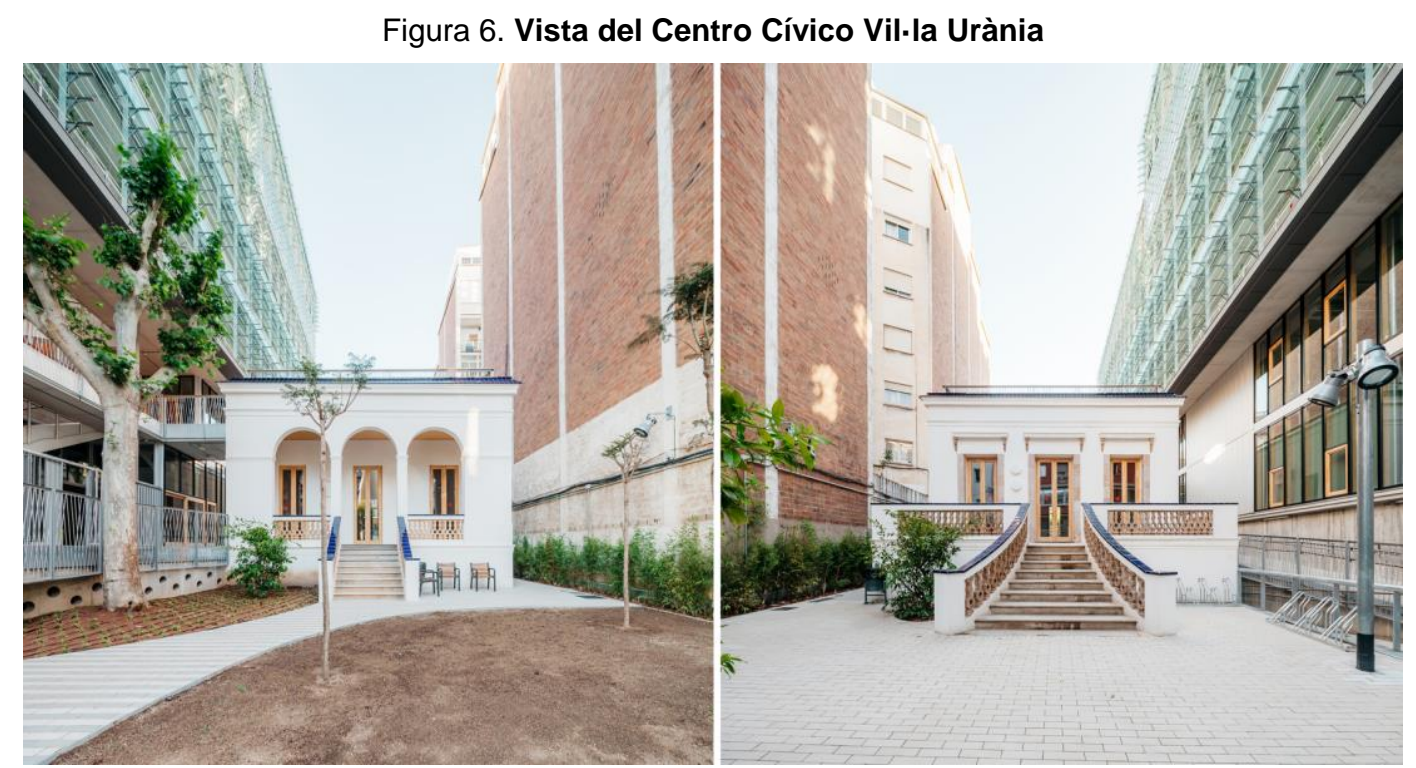

Fuente: SUMO arquitectes SLP, Fotógrafo: Aitor Estévez Olaizola 


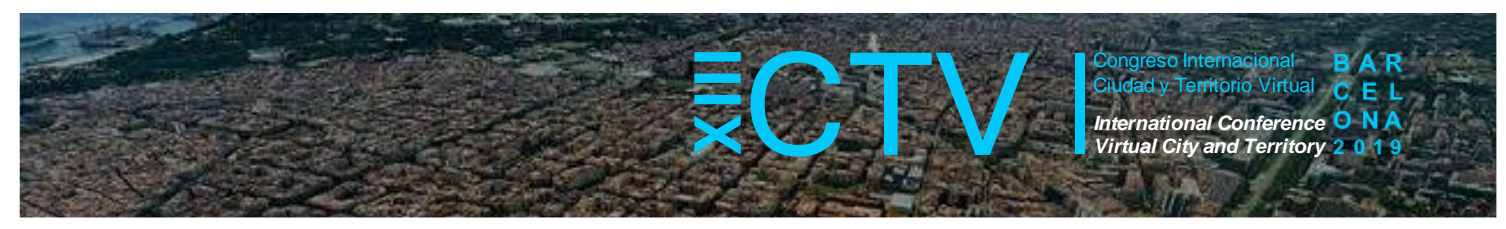

Figura 7. Vista desde la calle del Centro Cívico Vil·la Urània
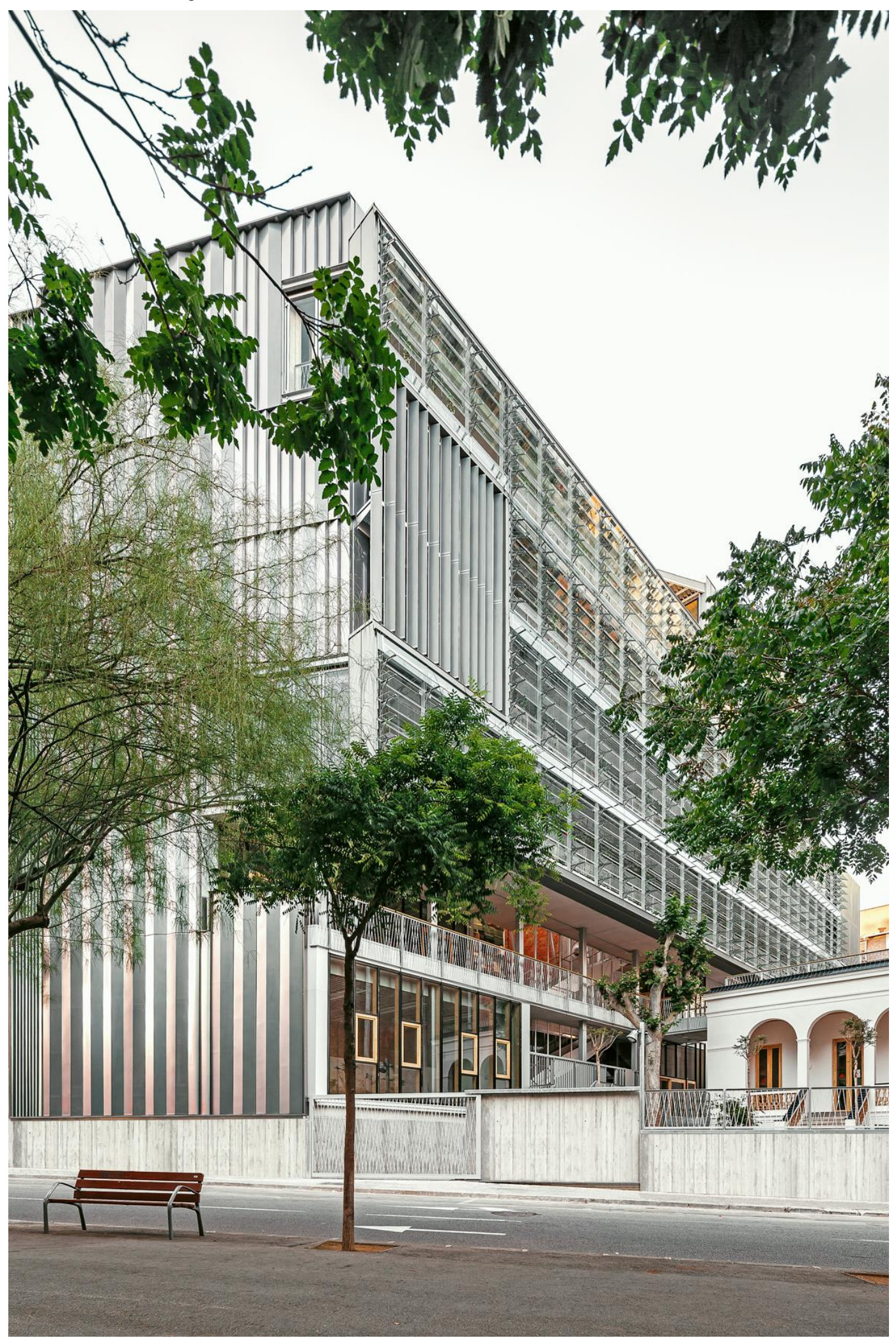

Fuente: SUMO arquitectes SLP, Fotógrafo: Aitor Estévez Olaizola. 


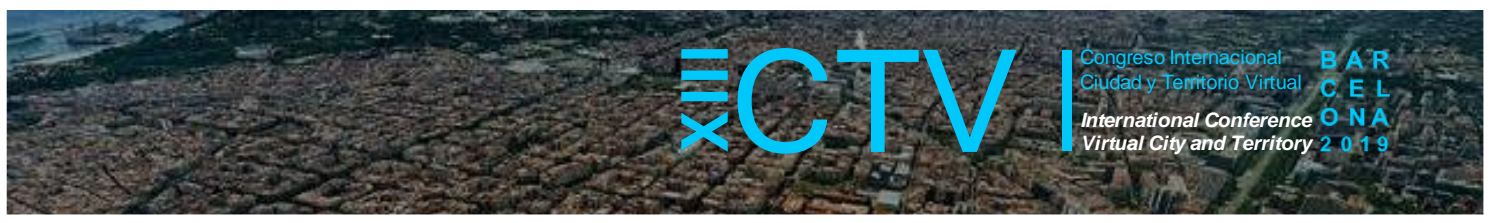

Figuras 8 y 9 . Vista interior del espacio intermedio
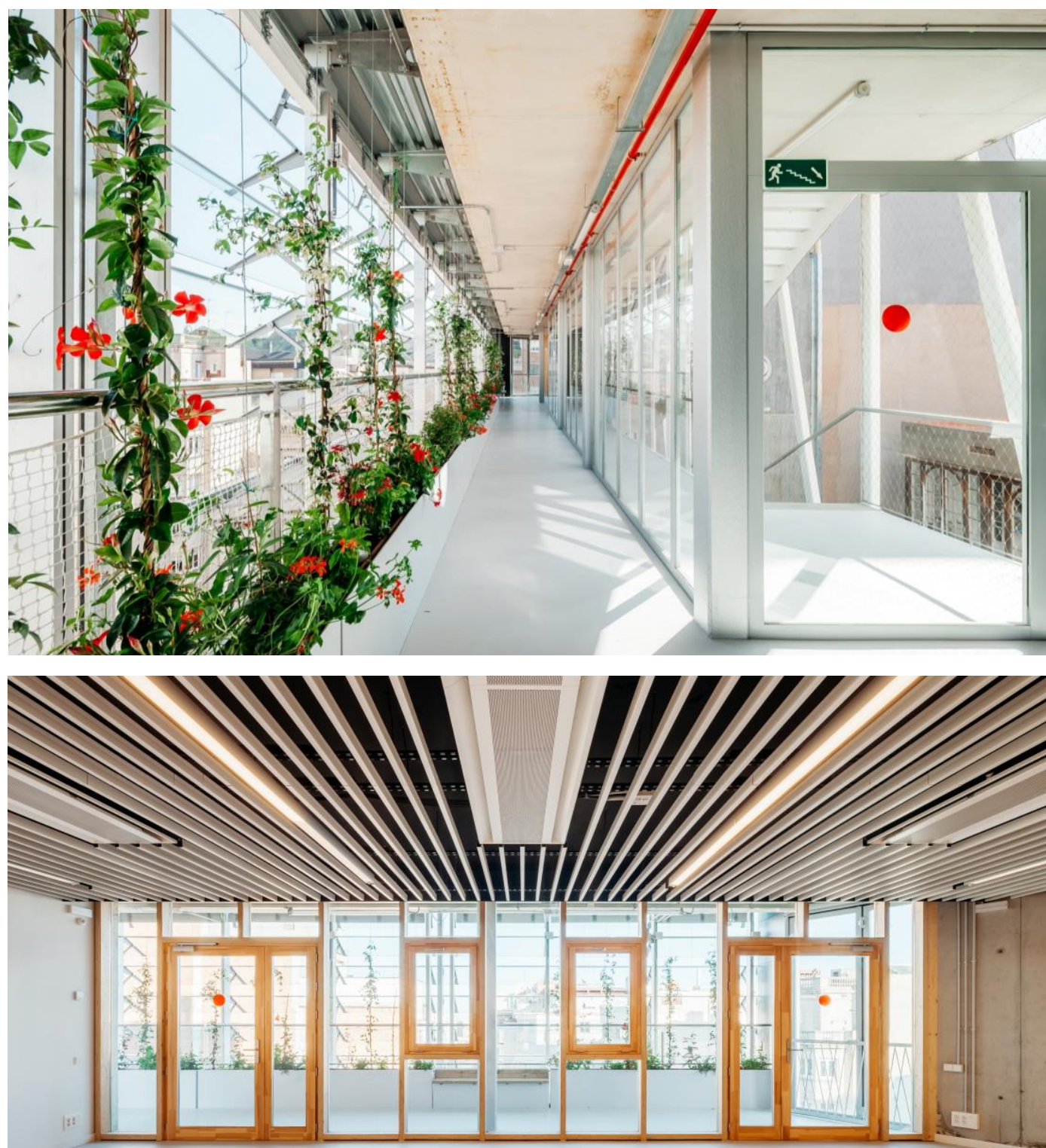

Fuente: SUMO arquitectes SLP, Fotógrafo: Aitor Estévez Olaizola

Contribuciones de los autores: Los autores han trabajado el documento de manera conjunta.

Conflicto de Intereses: Los autores declaran que no hay conflicto de intereses.

\section{Bibliografía}

Casaldàliga,Pau; Ripoll, Patricia; Pich-Aguilera, Felipe; Batlle, Teresa (2017) Edificis de consum d'energia gairebé zero. Barcelona, España: ICAEN 